\title{
Fatigue design and testing of automotive stabilizer bars
}

\author{
Efstratios Giannakis ${ }^{1,{ }^{*}}$, Elli Sivena ${ }^{1}$, Michail Malikoutsakis ${ }^{1}$ and Georgios Savaidis ${ }^{1}$ \\ ${ }^{1}$ Aristotle University of Thessaloniki, Laboratory of Machine Elements and Machine Design, 54124 \\ Thessaloniki, Greece
}

\begin{abstract}
Stabilisers are components of the axle suspension system of cars trucks, trains and other moving road vehicles that connect the movement of the two sides of the suspension (right and left) to keep the vehicle levelled. Stabilizers experience bending and torsion at operation. In particular, their arms experience bending while the main body mainly torsional loading. In both cases, the highly stressed area is the surface, where the maximum tensile and shear stresses are acting. High strength steels and special treatments, thermal and/or mechanical, are used for the stabilisers' manufacturing. The present study deals with necessary input data for fatigue life assessments based on the FKM guideline [1]. In addition, fatigue tests are conducted to calculate the stress-life curves of two different manufacturing processes.
\end{abstract}

\section{Introduction}

Automotive industry is always pushing the limits to have lighter and stronger structural components to reduce net weight, production costs and emissions. Thus, high strength steels and special treatments, thermal and mechanical, are used to manufacture light components that are robust enough to withstand a vehicle's lifetime. To make such an optimized component the designer has to take into account the manufacturing process and quantify every individual effect mostly using guidelines [2]. Particularly, in the case of the stabilizers, figure 1, high strength steels are used and undergo either hot morphing, quenching and tempering and shot peening, or thermal treatment, cold forming and shot peening to reach the specified endurance [3]. The stabilizers treated in the present study are manufactured with the second manufacturing procedure.

\footnotetext{
* Corresponding author: efstratg@auth.gr
} 


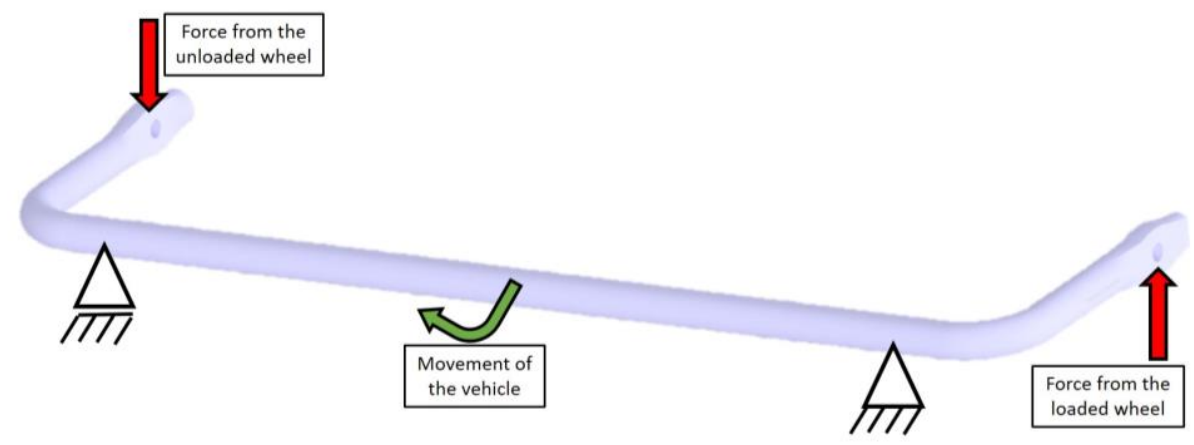

Fig. 1. Stabiliser under investigation and loading configuration under normal operation.

Except for the initial thermal treatment, in which the final microstructure is created throughout the stabiliser, and can be treated as the nominal material, the rest processes have both beneficial and detrimental attributes affecting that life. Cold morphing leaves residual stresses due to plastification of the area. Shot peening is the most common process, used to enhance surface fatigue properties, as it introduces compressive residual stresses [4] that lower the mean stress during operational fatigue loading. Despite being so advantageous, shot peening introduces a rough surface to the component which makes easier the crack initiation mechanism, reducing fatigue life. These two effects should be comprehended to reassure the positive effect of the procedure.

In this paper the effects of each process are discussed in two different types of specimens of the same geometry. These two types are analysed and tested. Firstly, the analysis of the microstructure in both configurations show the effects of the above mentioned thermal treatments, as well as the differences between them. Micro- and macrohardness is measured to quantify the mechanical properties of the materials which come out of the thermal treatment. In addition, the roughness of the surfaces and the residual stresses are to be considered within a theoretical fatigue life calculation.

A series of fatigue experiments is conducted to calculate the experimental stress life (SN) curves. Fatigue tests are performed on both types of specimens covering three stress levels. The S-N curves are elaborated for various probabilities of survival after statistical analysis.

Furthermore, the procedures presented in this paper demonstrate the way of fatiguebased stabiliser design, by reducing costs and the time needed to introduce a new stabiliser bar to the market, and also reducing the energy and effort for time-consuming fatigue experiments similarly to [5]. In addition, suggestions for future research on the field are stated.

\section{Material}

For better understanding of the specimens' strength performance, investigation on the material characteristics of both types has been performed. Metallographic analysis followed by hardness measurements reveal the effects of thermal treatment in both types while highlighting the differences in the microstructure.

\subsection{Microstructure}

To capture images of the microstructure few specimens of both batches were chosen and prepared. Additionally, two areas of interest were investigated, concerning the two stages of 
thermal treatment, the quenching and tempering and annealing. The first one focuses on the effects of thermal treatment to the component's core which reveals the material's reference characteristics. The second one focuses on the highly stressed area of the specimens, their surface, which is affected most by all the treatments.

The specimens' core microstructures can be seen in figures 2 and 3 in 500x and 1000x magnification, respectively for type 1 and 2 .
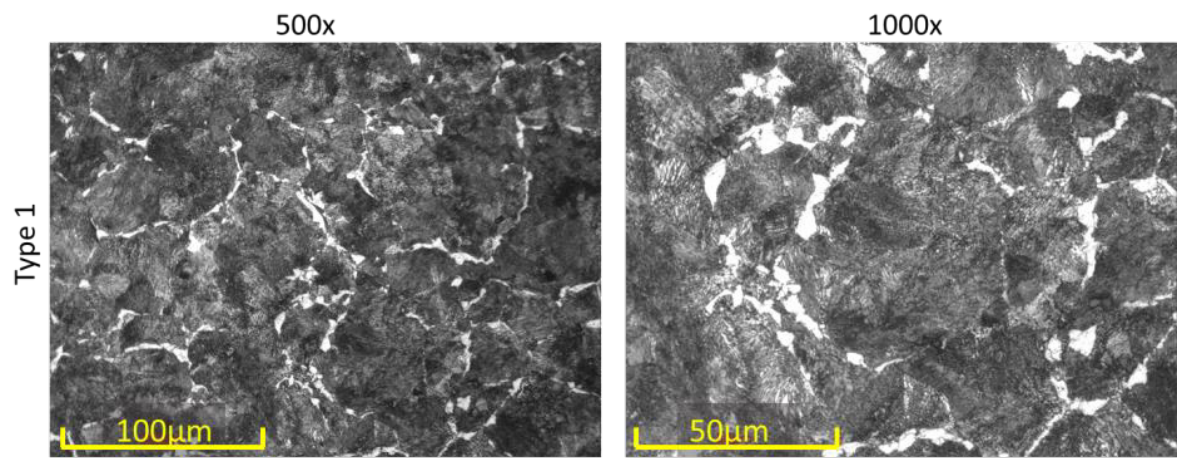

Fig. 2. Microstructure of type 1 specimen's core in 500x and 1000x magnification respectively.

Type 1 mainly consists of pearlite microstructure suggesting quenching with slow rate of temperature decrease [6]. Grain size is approximately $25 \mu \mathrm{m}$.
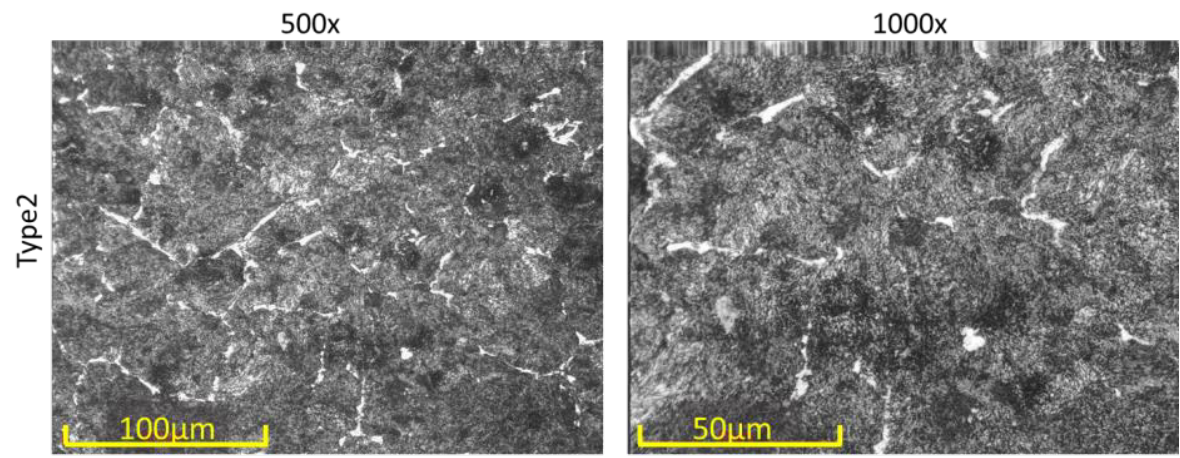

Fig. 3. Microstructure of type 2 specimen's core in 500x and 1000x magnification respectively.

Type 2 has the same microstructure as Type 1 .

Concerning the specimens' surface and the effects of thermal treatment, comparison figures of surface and core microstructure are shown in figures 4 and 5.
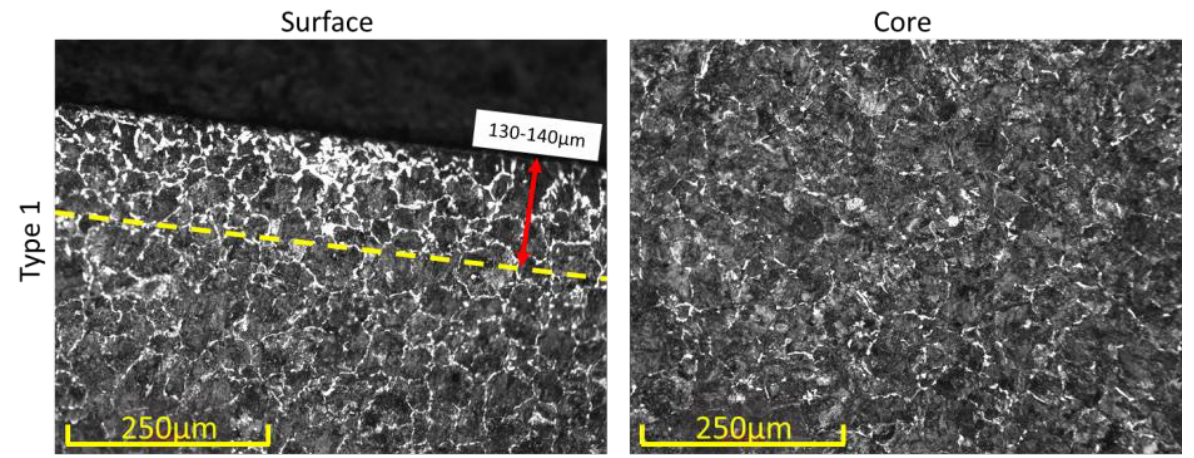
Fig. 4. Microstructure comparison of type 1 specimen's surface and core structures in 200x magnification.

Type 1 has a decarburization zone of approx. 130 to $140 \mu \mathrm{m}$ from the surface due to the annealing stage of manufacturing. This zone is easily recognizable as carbon is less.
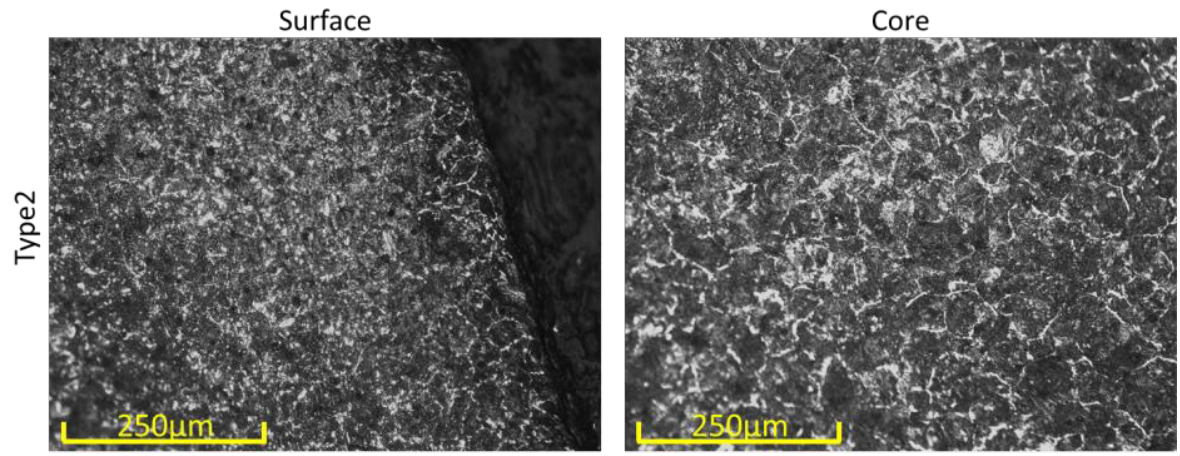

Fig. 5. Microstructure comparison of type 2 specimen's surface and core structures in 200x magnification.

On the other hand, type 2 does not have a decarburization zone suggesting a tougher surface, therefore, stronger material.

\subsection{Micro-hardness}

Vickers micro-hardness measurements show the hardness distribution from the surface towards the core. Notice that the microstructure observed in the metallographic analysis suggests a difference in the Vickers values between the two types. The hardness profiles can be seen in figure 6 for both types.

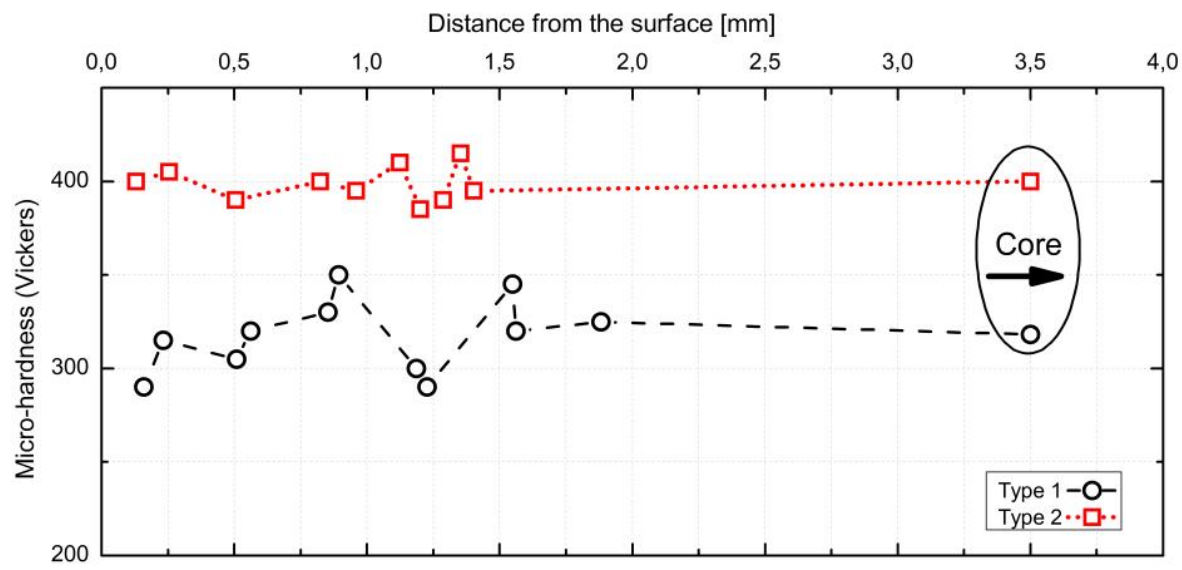

Fig. 6. Vickers micro-hardness profile.

In the first $1 \mathrm{~mm}$ of depth, there is a clear trend of higher hardness in Type 2 specimens that continues towards the core of the specimens. It has to be highlighted that Type 2 has almost constant hardness from the surface to the core, while Type 1 presents lower hardness values at the surface and towards the core. Having in mind that the grains have a size of approx. $25 \mu \mathrm{m}$ and the indentation has a diameter of about $50 \mu \mathrm{m}$ it is very difficult to 
understand the material's properties only using micro hardness measurements. Therefore, macro hardness tests were also performed.

\subsection{Macro-hardness}

Macro-hardness measurements are performed for better characterization of the material. Figure 7 shows the Rockwell C hardness from surface to core.

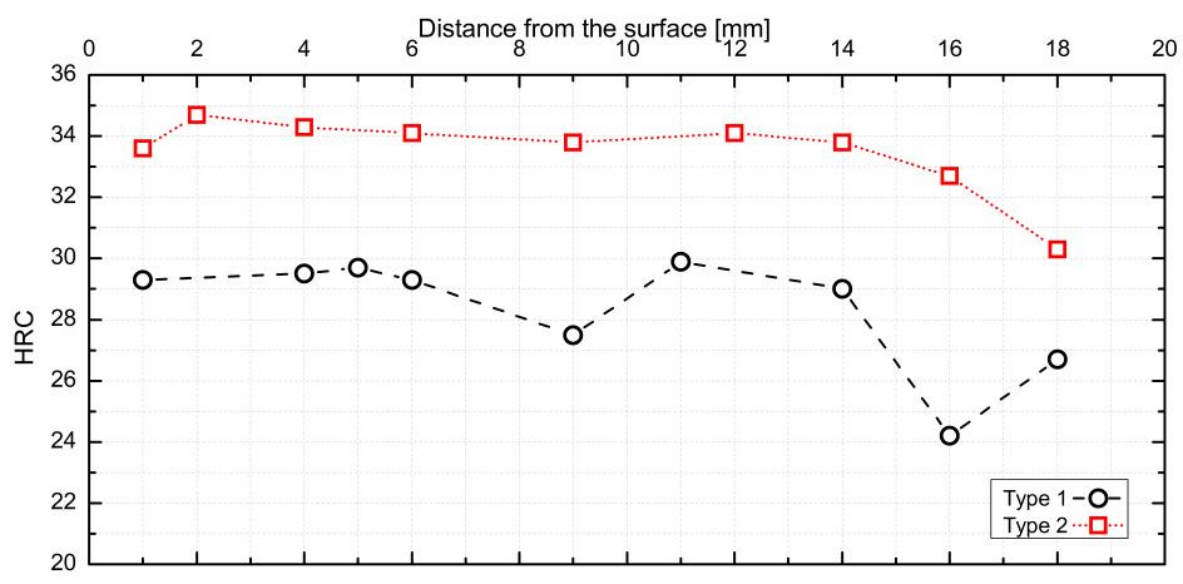

Fig. 7. HRC hardness measurements.

In contrast with the micro-hardness measurements the values are more stable and less affected by the structure's grains. Type 1 is harder than type 2 throughout the thickness of the specimen, suggesting a stronger material structure.

\subsection{Material assessment}

Notice that the two types exhibit approximately the same microstructure, judging by the metallographic analysis. A big difference is observed at the surface of the specimens, where Type 2 is harder, as there is no decarburization zone. The hardness measurements also suggest that Type 2 has a stronger structure overall.

\section{Surface treatments}

Both stabilizer types have been shot peened to improve their fatigue characteristics. Shot peening on the one hand introduces advantageous compressive residual stresses at the failure critical area, on the other hand the roughness created by the shots to the surface of the components affects negatively its fatigue endurance. The influence of these two effects can be calculated using available Guidelines like FKM.

\subsection{Roughness}

The roughness measurements of the specimens' surface are shown in table 1.

Table 1. Roughness values and roughness factor.

\begin{tabular}{|c|c|c|}
\hline Roughness & Type 1 & Type 2 \\
\hline
\end{tabular}




\begin{tabular}{|c|c|c|}
\hline $\mathrm{Rz}$ & 9.8125 & 23.1875 \\
\hline $\mathrm{Ra}$ & 1.58 & 4 \\
\hline $\mathrm{K}_{\mathrm{R}, \sigma}$ & 0,84 & 0,79 \\
\hline
\end{tabular}

The values show that type 2 has a rougher surface than type 1, resulting in a lower roughness factor that has negative effect to the fatigue performance.

\subsection{Residual stresses}

According to the FKM Guideline the positive effect of the compressive residual stresses is limited to a factor without taking into account their intensity. The surface treatment factor is calculated as $\mathrm{K}_{\mathrm{V}}=1.1-1.3$.

\section{Fatigue experiments}

Fatigue experiments have been performed to both stabilizer types at the same loading levels. For type 1, fourteen results are used to calculate the S-N curve and for Type 2 five results that are available at the time of the present paper submission and two specimens that have not fractured yet.

All experiments are set using strain gages and tested at three loading levels. In figure 8, the normalized stress life curves of type 1 and all the experimental results are presented. Even though only five type 2-specimens fractured and two are still running, clear tendency of better fatigue performance of Type 2 is visible. More experiments are necessary to draw safer conclusions and a stress life curve for Type 2.

\section{Experiments Comparison}

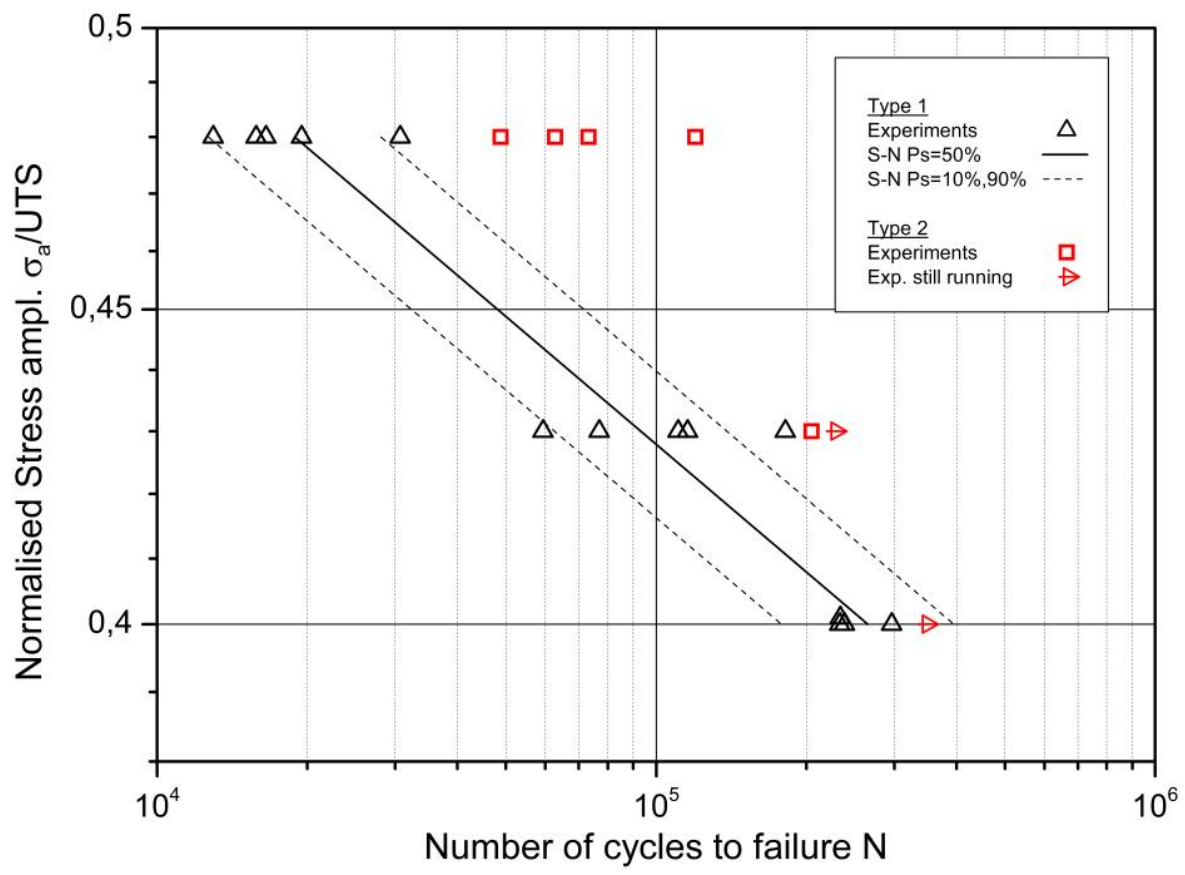

Fig. 8. Comparison of fatigue life results of Type 1 and 2 at fully reversed loading $R=-1$. 


\section{Summary and conclusions}

In this investigation two types of stabilisers were tested and analysed. They provide the same geometry and material, and experienced different manufacturing process steps. Through metallographic analysis, hardness and roughness measurements, data is gathered in addition to fatigue experiments for the calculation of typical stress life curves. The two types of stabilisers have differences in the microstructure of the failure critical, different hardness and roughness on the surface. Although type 1 stabilisers have a very smooth surface, its thermal treatment is less effective as it is less hard than type 2 which also has a stronger structure in the critical failure area. Though the performed experiments are only 14 for Type 1 and 7 for Type 2, Type 2 seems to have longer endurance than Type 1. All gathered data will be used on the development of a theoretical model based on the FKM guideline.

\section{References}

1. B. Hänel, E. Haibach, T. Seeger, G. Wirthgen, H. Zenner, 5th Edition translated by E. Haibach, VDMA-Verlag, Frankfurt, (2003).

2. E. Giannakis, G. Savaidis, Materials Science and Technology, WILEY-VCH Verlag GmbH \& Co. KGaA, vol 47, part 10, pp 897-903, Weinheim, September, (2016).

3. SAE Spring Committee, 2nd Edition, vol. 3, Society of Automotive Engineers, (1996).

4. E. Müller, Metal Finishing News, Volume 5, Issue May, Wetzikon (2004).

5. E. Giannakis, M. Malikoutsakis, G. Savaidis, Innovative Manufacturing Engineering and Energy International Conference (IMANEE) 2016, IOP Conference Series: Materials Science and Engineering, vol 161, N. 1, Greece, December, (2016).

6. G. D. Chrysoulakis, D. I. Pantelis, Epistimi kai Technologia ton Metallikon Ylikon. 2nd Edition, Papasotiriou, Athens, (2008). 